\title{
Direct CCL4 Inhibition Modulates Gut Microbiota, Reduces Circulating Trimethylamine N-Oxide, and Improves Glucose and Lipid Metabolism in High-Fat- Diet-Induced Diabetes Mellitus
}

\author{
Ting-Ting Chang ${ }^{\mathrm{I}-3}$ \\ Jaw-Wen Chen ${ }^{1-6}$ \\ 'Department and Institute of \\ Pharmacology, School of Medicine, \\ National Yang-Ming University, Taipei, \\ Taiwan; ${ }^{2}$ Department and Institute of \\ Pharmacology, National Yang Ming Chiao \\ Tung University, Taipei, Taiwan; ${ }^{3}$ School \\ of Medicine, National Yang Ming Chiao \\ Tung University, Taipei, Taiwan; \\ ${ }^{4}$ Healthcare and Services Center, Taipei \\ Veterans General Hospital, Taipei, \\ Taiwan; ${ }^{5}$ Division of Cardiology, \\ Department of Medicine, Taipei Veterans \\ General Hospital, Taipei, Taiwan; \\ ${ }^{6}$ Cardiovascular Research Center, \\ National Yang Ming Chiao Tung \\ University, Taipei, Taiwan
}

Purpose: Modulation of the gut microbiota may lead to changes in pathological conditions. C-C chemokine motif ligand (CCL) 4 was upregulated in diabetes mellitus (DM) and was shown to play a significant role in pancreatic inflammation and glucose metabolism. The detailed in vivo mechanisms have not been well explored. This study aimed to investigate the hypothesis that direct CCL4 inhibition could modify gut microbiota and systemic metabolism in diet-induced DM mice.

Methods: C57BL/6 mice fed a high-fat diet (HFD) were used as a diet-induced DM model. CCL4 inhibition was conducted by anti-CCL4 neutralizing monoclonal antibodies. The gut microbiota was analyzed by high-throughput sequencing of the 16S rRNA. Fecal microbiota transplantation (FMT) was used to verify the effect of CCL4 deficiency on gut microbiota and the linkage between CCL4-modulated gut microbiota and HFD-induced DM.

Results: CCL4 inhibition stabilized glucose homeostasis, modulated lipid parameter, and decreased inflammatory markers in HFD-induced DM mice. Moreover, CCL4 inhibition reversed HFD-induced gut dysbiosis, evidenced by the decreased abundance of family Muribaculaceae and increased abundance of family Atopobiaceae when CCL4 antibodies were administrated. CCL4 inhibition led to a decrease in circulating trimethylamine N-oxide levels, a proinflammatory metabolite from gut microbiota. Taken together, CCL4 inhibition could modify gut microbiota profiles, suppress proinflammatory metabolites, reduce systemic inflammation, improve insulin resistance, and retard the progression of hyperglycemia in HFD-induced DM. Furthermore, FMT from CCL4 knockout mice rescued the glucose homeostasis in HFD-induced DM mice.

Conclusion: Our findings may not only provide a novel rationale to in vivo CCL4-based therapeutic approach in diet-induced DM but also indicate the significance of gut microbiota profile including the family Muribaculaceae and the family Atopobiaceae as a potential modifiable target for systemic metabolism.

Keywords: diabetes mellitus, gut microbiota, inflammation, chemokine, trimethylamine $\mathrm{N}$-oxide

\section{Introduction}

The gut microbiota affects pathological conditions including obesity, diabetes mellitus (DM), and cardiovascular disease. ${ }^{1,2}$ Previous studies indicated that the gut microbiota may play a potential role in interfering with host metabolism, enhancing the risk factors of metabolic syndrome. Several micro-organism-derived metabolites, such as 


\section{Graphical Abstract}

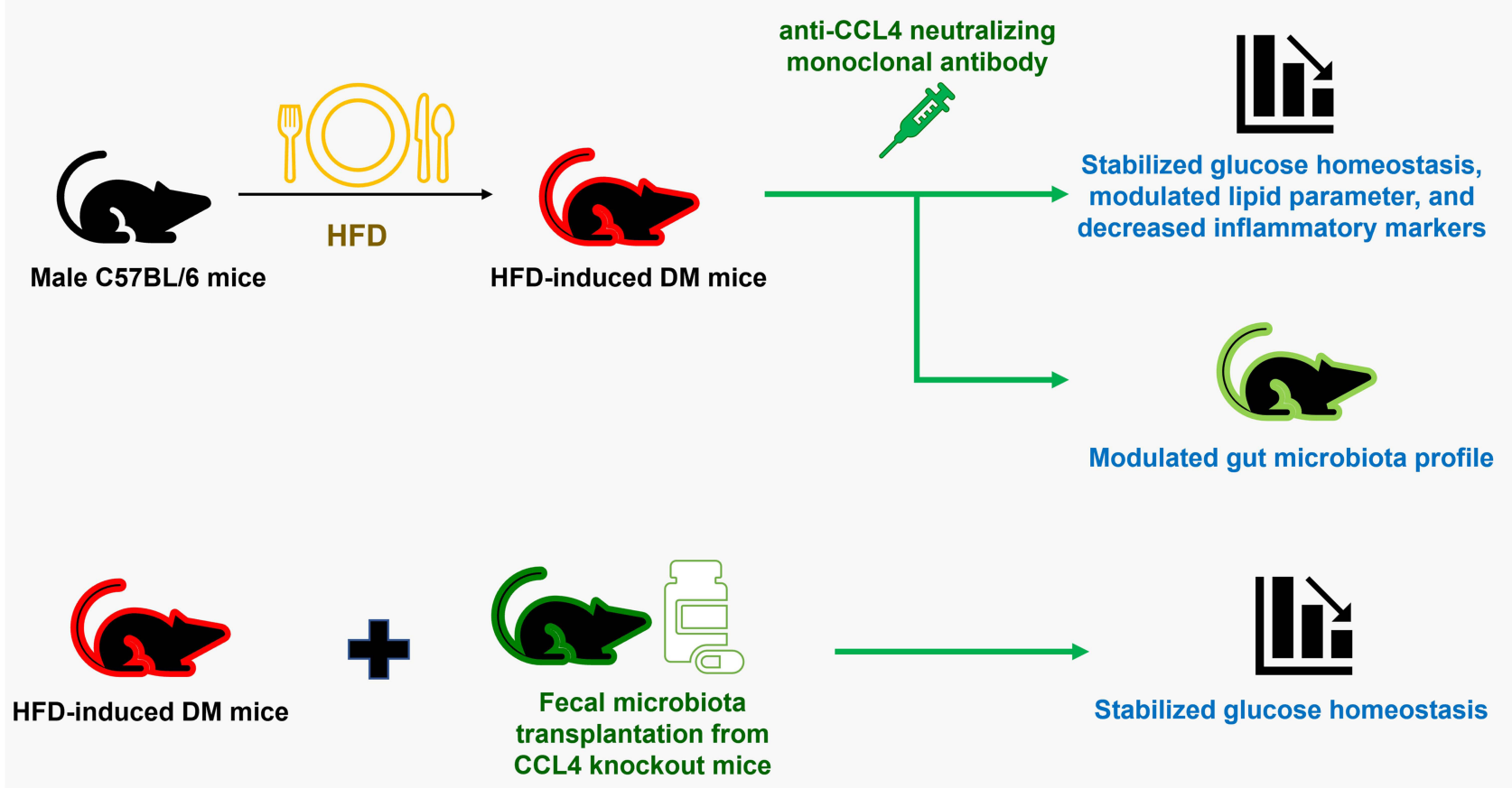

trimethylamine N-oxide (TMAO), lipopolysaccharide (LPS), indoxyl sulfate, and p-cresol sulfate, could activate the inflammatory processes in metabolic syndrome. ${ }^{3}$ Systemic inflammation has been shown to be associated with the progression of hyperglycemia., ${ }^{4,5}$ Some diets could alter the gut microbiota, leading to changes in the lipid profile and accelerated systemic inflammation. ${ }^{6,7}$ Antidiabetic drugs and food supplementation could interfere with the gut microbiota to improve glucose tolerance and reduce inflammation in a high-fat diet (HFD)-induced diabetic mouse model, ${ }^{8,9}$ indicating the relationship between pathophysiological factors and the gut microbiota. More investigations of the role of the gut microbiota in dietinduced diabetes might provide new therapeutic approaches.

The levels of C-C chemokine motif ligand (CCL) 4 are similarly elevated in both type 1 and type 2 DM patients, implying that CCL4 might be related to systemic inflammation in different types of DM as well. ${ }^{10,11}$ In an HFDinduced diabetic mouse model, isoliquiritigenin-treated mice had higher plasma cholesterol and improved insulin resistance, with decreased CCL4 and proinflammatory levels of cytokines including interleukin (IL)-1 $\beta$, IL-6, and monocyte chemoattractant protein-1. ${ }^{12}$ Furthermore, a diet including carotenoid-rich vegetables and fruits affects inflammation by decreasing CCL4 and tumor necrosis factor (TNF)- $\alpha$ levels in healthy individuals. ${ }^{13}$ Our previous study indicated that inhibition of CCL4 could control blood sugar levels, increase serum insulin levels, and decrease pancreatic IL-6 expression in streptozotocin-induced diabetic mice. Meanwhile, CCL4 inhibition could retard the progression of hyperglycemia, reduce serum tumor necrosis factor (TNF)- $\alpha$ and IL-6 levels, and improve insulin resistance in mice with type $2 \mathrm{DM}$ or metabolic syndrome. These results suggested the critical role of CCL4-related inflammation in the progression of DM. ${ }^{14}$ However, the in vivo effects of CCL4 inhibition are complex and the potential mechanisms have not been completely elucidated. The evidence is still lacking regarding the potential effects of CCL4 on gut microbiota, which has been linked to the presence of systemic inflammation and insulin resistance in diet-induced diabetes.

This study aimed to investigate the hypothesis that direct CCL4 inhibition could modify gut microbiota and related systemic metabolism in diet-induced DM mice. In this study, the HFD-induced diabetic mouse model was used to investigate whether targeting on CCL4 inhibition could directly modify gut microbiota profile, reduce systemic inflammation, improve insulin resistance, and retard 
the progression of hyperglycemia. Then, fecal microbiota transplantation (FMT) was performed to prove our concept that the gut microbiota mediated by CCL4 could rescue the pathology in diet-induced DM. Our findings may not only provide additional novel rationale to in vivo CCL4based approach but also hopefully identify some specific gut microbiota related to metabolic control in diet-induced DM.

\section{Materials and Methods}

\section{Animal Procedures}

Six-week-old male C57BL/6 mice were purchased from the National Laboratory Animal Center (Taipei, Taiwan). LabDiet Rodent 5001 (23.9\% protein, 5\% fat (ether extract), $5 \%$ fat (acid hydrolysis); LabDiet, St. Louis, MO, USA) was used as a normal diet. C57BL/6 mice were fed a high-fat diet (23.6\% protein, $34.9 \%$ fat, $25.9 \%$ carbohydrate, diet-induced obesity rodent-purified diet with $60 \%$ energy from fat-58Y1) for 14 weeks. Blood glucose was evaluated after the mice were fasted for 4 hours.

In another part of the experiments, CCL4 knockout (C57BL/6JNarl-Ccl4eml) mice were designed and generated via the CRISPR/Cas9 system by the National Laboratory Animal Center (Taipei, Taiwan). C57BL/6 mice were purchased from the National Laboratory Animal Center (Taipei, Taiwan) as the wild-type control group. The mice were sacrificed in deeply anesthesia induced via inhaled isoflurane. Animal experiments were conducted at National Yang Ming Chiao Tung University. Animals were raised according to the regulations of the Animal Care Committee of National Yang Ming Chiao Tung University. All animal-related work was performed under the Institutional Animal Care and Use Committee (IACUC) protocol approved by National Yang Ming Chiao Tung University (IACUC no. 1031275). Some diet-induced diabetic mice received an intraperitoneal injection of an anti-CCL4 neutralizing monoclonal antibody (MAB451, $100 \mu \mathrm{g}$; R\&D Systems, Minneapolis, MN, USA) 3 times per week for 2 or 4 weeks. The rat $\operatorname{IgG}_{2 \mathrm{~A}}$ isotype (MAB006, $100 \mu \mathrm{g}$; R\&D Systems) was administered as a control. Body weights and blood sugar concentrations were measured.

\section{Fecal Microbiota Transplantation (FMT)}

Antibiotic water containing ciprofloxacin $0.2 \mathrm{~g} / \mathrm{L}$ were given before FMT for 2 days. FMT was conducted each weekday for 12 weeks. Feces were collected fresh from CCL4-deficient mice for daily FMT. Feces from CCL4- deficient mice were pooled by cage, and about $100 \mathrm{mg}$ (about 5-6 fecal pellets) were re-suspended in $1 \mathrm{~mL}$ sterile saline. Feces and saline solution were mixed for $10 \mathrm{sec}-$ onds, then centrifugation at $800 \mathrm{~g}$ for 3 minutes. The supernatants (about 500-600 $\mu \mathrm{L}$ ) were collected and administered by oral gavage within 10 minutes to minimize the change in microbial content.

\section{Oral Glucose Tolerance Tests (OGTTs) and Areas Under the Curves (AUCs)}

Mice were fasted for 6 hours and orally administered glucose at a dose of $2 \mathrm{~g} / \mathrm{kg}$ body weight. Blood samples were obtained at time 0 (just before glucose load) and at 15, 30, $60,120,150$, and 180 minutes after glucose administration. The total and incremental AUCs for plasma glucose during the OGTT were determined by the trapezoidal method.

\section{Homeostasis Model Assessment of Insulin Resistance (HOMA-IR)}

Insulin resistance, insulin sensitivity, and steady-state $\beta$ cell function were determined by the homeostasis model assessment of insulin resistance (HOMA-IR) using fasting glucose and insulin levels. HOMA analysis is an accepted surrogate for measuring insulin resistance in rodents. ${ }^{15}$ The HOMA-IR index was calculated as (fasting insulin $(\mu \mathrm{U} / \mathrm{mL}) \times$ fasting glucose $(\mathrm{mmol} / \mathrm{L})) / 22.5{ }^{16}$

\section{Biochemical Indices}

Serum levels of total cholesterol (TC), triglycerides (TG), and high-density lipoprotein (HDL) were determined after a $4 \mathrm{~h}$ fast using an automated clinical chemistry analyzer (FUJI DRI-CHEM 4000i). Blood glucose levels were measured by Optium Xceed.

\section{Evaluation of TNF- $\alpha$, IL- 6 and Insulin Concentrations}

Serum protein concentrations of TNF- $\alpha$ and IL-6 were determined by ELISA (R\&D Systems) according to the manufacturer's instructions. Serum concentrations of insulin were determined by ELISA (Millipore) according to the manufacturer's instructions.

\section{DNA Extraction and DNA Quality Control} We used a QIAamp ${ }^{\circledR}$ DNA Stool Mini Kit from QIAGEN (cat. no. 51504) to collect stool specimens. The quality and quantity of samples were checked by NanoPhotometer ${ }^{\mathrm{TM}}$ Pearl (Implen). 


\section{Library Construction and Sequencing for V3V4 Region of I6S Ribosomal DNA}

$16 \mathrm{~S}$ metagenomics library was constructed using two rounds of PCR reactions. ThePCRprimers, forward(5'TCGTCGGCAGCGTCAGATGTGTATAAGAGACAGCCTACGGGNGGCWGCAG-3') and reverse (5'- GTCT CGTGGGCTCGGAGATGTGTATAAGAGACAGGGACTACHVGGGTWTCTAAT-3'), were designed to amplify the V3V4 domain of bacterial 16S ribosomal DNA and add adaptor sequences. Illumina index sequences were subsequently attached to the amplicons in the second PCR. PCR amplification was performed in a $50 \mu \mathrm{L}$ reaction volume containing $25 \mu \mathrm{L} 2 \mathrm{X}$ Phusion High-Fidelity PCR Master Mix (New England Biolabs), $0.2 \mu \mathrm{M}$ of both the forward and reverse primer, and $100 \mathrm{ng}$ DNA template. The reaction conditions consisted of an initial $98{ }^{\circ} \mathrm{C}$ for 30 seconds, followed by 30 cycles of $98{ }^{\circ} \mathrm{C}$ for 10 seconds, $55^{\circ} \mathrm{C}$ for 30 seconds, $72{ }^{\circ} \mathrm{C}$ for 30 seconds, and $72{ }^{\circ} \mathrm{C}$ for 5 min. There were 10 cycles for the second PCR. Amplified products were then checked by $2 \%$ agarose gel electrophoresis and purified using the AMPure XP PCR Purification Kit (Agencourt). Quantification was performed using Qubit dsDNA HS Assay Kit (Thermo Fisher Scientific) on Qubit 2.0 Fluorometer (Thermo Fisher Scientific) and LightCycler 96 (Roche) according to the manufacturers' instructions. The final purified libraries were applied for cluster generation and sequencing on the MiSeq using a V3 600 cycles kit.

\section{Bioinformatics}

Sequencing reads were first quality controlled using FASTX-Toolkit with the following parameters: fastq quality_filter -Q33 -q 20 -p 70; and fastq_quality_trimmer -t 20 -1 200 -Q33. Then, UPARSE (https://www.drive5.com/ uparse/) was employed for OTU clustering using the reads that passed quality control. Bowtie2 (http://bowtie-bio. sourceforge.net/bowtie2/index.shtml) was used for taxonomic assignment to align OTUs with the SILVA database (v132). The alignment identity was set as $97 \%$. R package (v3.5.1) was employed to calculate relative abundance, abundance heatmap, alpha-diversity and beta-diversity.

\section{Measurement of Serum Trimethylamine $\mathrm{N}-$ Oxide (TMAO)}

For each sample, $50 \mu \mathrm{L}$ of blood was obtained to measure the TMAO after fasting for 6 hours. After the blood sample was centrifuged $(3000 \times \mathrm{g}$ for 15 minutes at 4
${ }^{\circ} \mathrm{C}$ ), all serum samples were stored at $-80{ }^{\circ} \mathrm{C}$ until the TMAO measurement. In detail, $50 \mu \mathrm{L}$ of serum was mixed with $450 \mu \mathrm{L} \mathrm{MeOH}$ and TMAO-d9 isotopologues. After preparation, serum TMAO was analyzed by injection into a MicroSolv Cogent Diamond Hydride column (150 × $2.1 \mathrm{~mm}, 4.2 \mu \mathrm{m}$, MicroSolv, Eatontown, NJ, USA) interfaced with an Agilent 1290 UHPLC and coupled with an Agilent 6460 triple quadrupole mass spectrometer (Agilent Technologies, Santa Clara, CA). Precursor-product ion transitions occurred at $\mathrm{m} / \mathrm{z} \quad 76.1 \rightarrow 58.1$ and $76.1 \rightarrow 59.1$ (TMAO), $\mathrm{m} / \mathrm{z} \quad 85.1 \rightarrow 66.3$, and $85.1 \rightarrow 68.3$ (TMAO-d9). The results were represented as fold of the HFD group.

\section{Statistics}

Results are presented as the mean \pm standard error of the mean (SEM). Statistical analyses were performed using unpaired Student's $t$-tests or analysis of variance, followed by Scheffe's multiple-comparison post-hoc test. SPSS software (version 14; SPSS, Chicago, IL, USA) was used to analyze data. A $p$-value $<0.05$ was considered statistically significant.

\section{Results \\ CCL4 Inhibition Provided Beneficial Effects on Glucose Homeostasis}

C57BL/6 mice were fed a high-fat diet to mimic the clinical development of diet-induced DM. The body weights were increased in the HFD-induced DM mice compared to those fed a normal diet. CCL4 antibody injections did not affect the body weight of HFD-induced DM mice (Figure 1A). The blood sugar levels were increased in HFD-induced DM mice compared to mice fed a normal diet. Blood sugar levels were decreased in the HFD-induced DM mice that received the CCL4 antibody injection for 4 weeks (Figure 1B). In the OGTT test, blood sugar levels were lower in the CCL4 antibody-treated group at 15, 30, 60 and 120 minutes than in the untreated HFD-induced DM group (Figure 1C). The CCL4 inhibition group also had lower levels of HOMA-IR than the HFD-induced DM group (Figure 1D). These results suggested that CCL4 inhibition had beneficial effects on glucose homeostasis in HFD-induced DM mice.

\section{CCL4 Inhibition Modulated Lipid}

\section{Parameter and Inflammation in HFD-Induced DM Mice}

Due to the close relationship between diet-induced obesity and dyslipidemia, we also investigated the serum lipid profile in mice. HFD-induced DM mice showed 
A

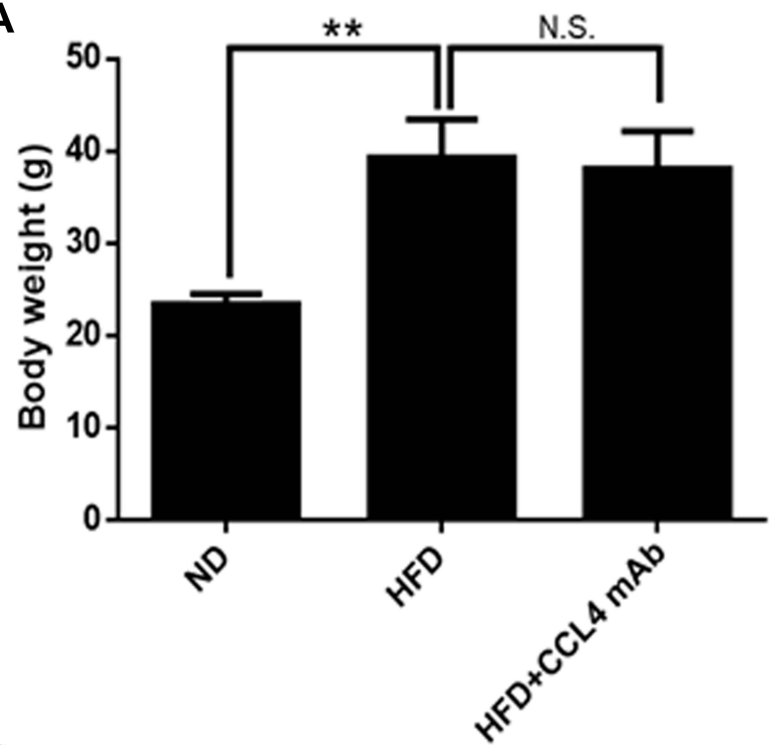

C

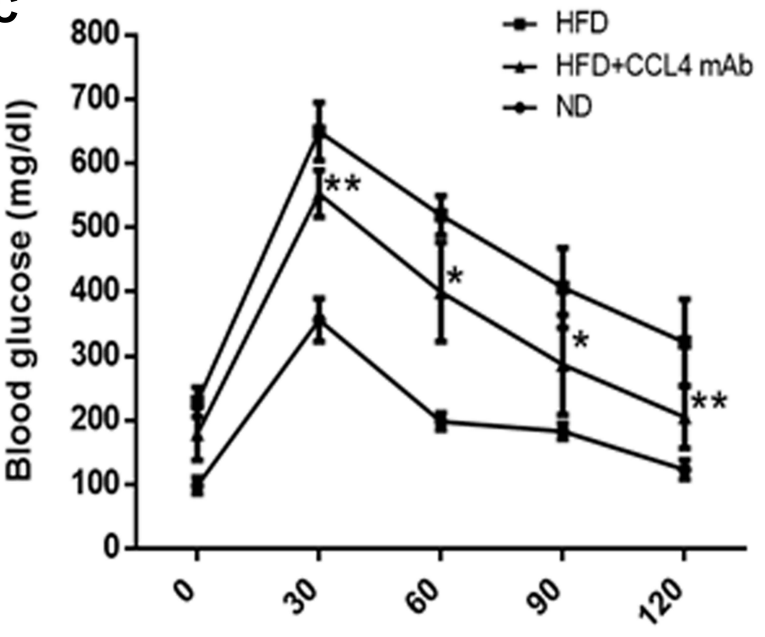

Time (minutes)
B

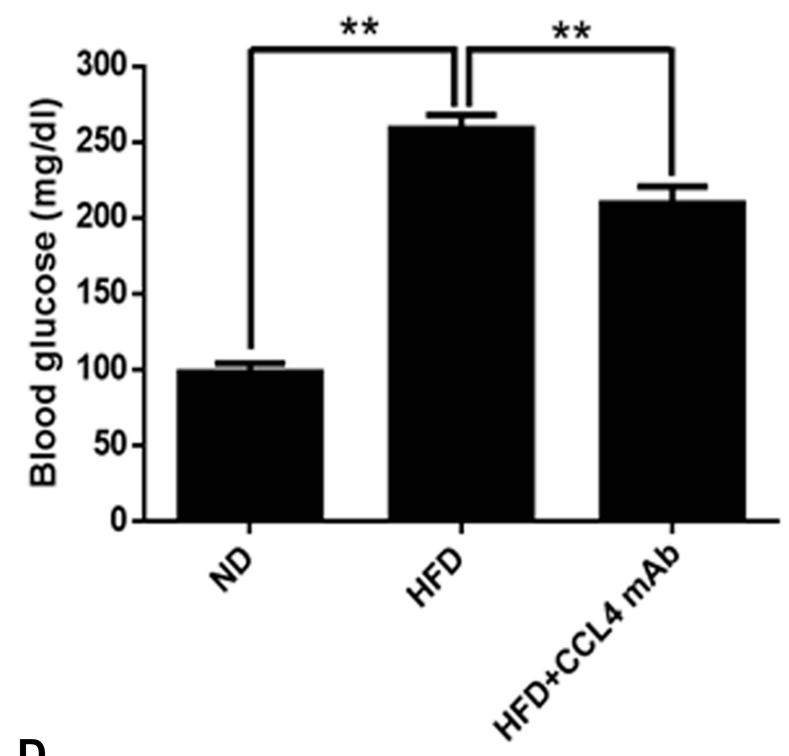

D

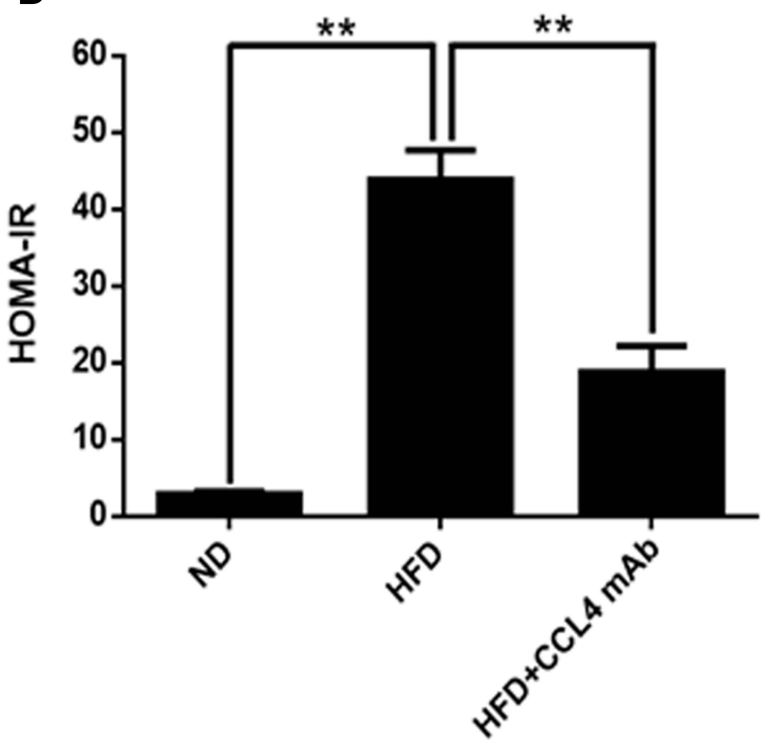

Figure I The effect of CCL4 inhibition on glucose homeostasis and systemic inflammation in HFD-induced DM mice. Body weights and blood sugar levels ( $\mathrm{n}=6$; (A and B)). The oral glucose tolerance tests (OGTTs) and homeostasis model assessment of insulin resistance (HOMA-IR) were conducted after CCL4 antibody injections for 2 weeks $(n=6 ;(C$ and $\mathbf{D})$ ). ND represents normal diet mice; HFD represents HFD-induced DM mice. *P $<0.05$, **P $<0.01$.

Abbreviation: NS, not significant.

dyslipidemia symptoms, as evidenced by the increased total cholesterol (TC), triglycerides (TG), and non-highdensity lipoprotein (HDL) levels compared to mice fed a normal diet. The CCL4 inhibition group exhibited decreased serum TC, TG, and non-HDL levels compared to the untreated HFD-induced DM group (Figure 2A-C). Moreover, obesity was characterized by systemic and tissue inflammation. ${ }^{17}$ The serum TNF- $\alpha$ and IL-6 levels were enhanced in the HFD-induced DM group compared to those fed a normal diet. The enhanced inflammatory markers were both reversed after CCL4 antibody injection for 4 weeks (Figure 2D and E). These results indicated that CCL4 inhibition was able to modulate the lipid profile and reduce systemic inflammation in HFD-induced DM mice.

\section{CCL4 Inhibition Changed the Structure} of Gut Microbiota in HFD-Induced DM

\section{Mice}

We hypothesized that CCL4 inhibition plays a critical role in programming host-microbe metabolic interactions and 

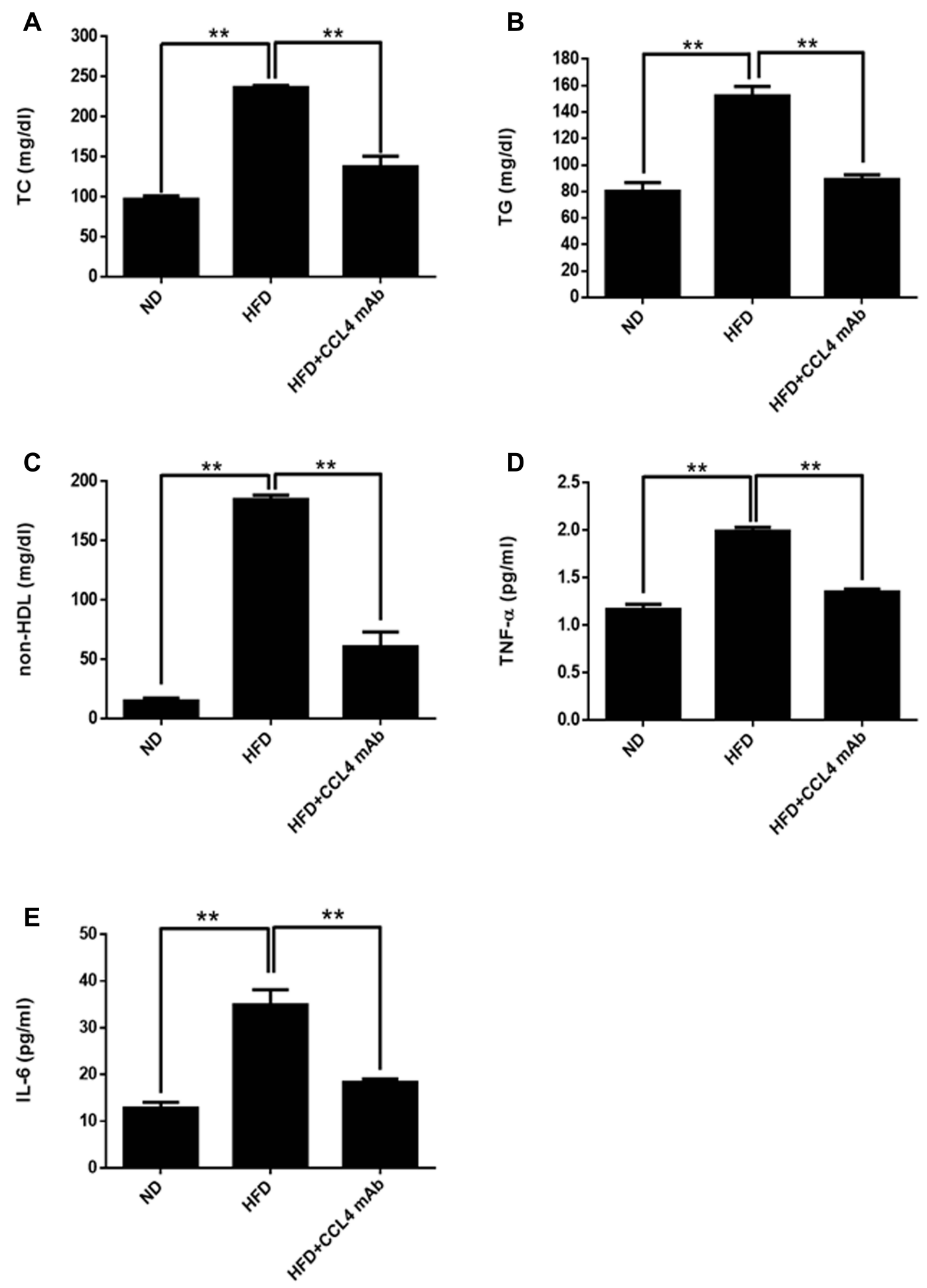

Figure 2 The effect of CCL4 inhibition on lipid parameter and systemic inflammation in HFD-induced DM mice. Serum total cholesterol (TC), triglycerides (TG), and nonhigh-density lipoprotein $(H D L)$ levels $(n=6 ;(\mathbf{A}-\mathbf{C}))$. Serum tumor necrosis factor $(T N F)-\alpha$ and interleukin $(\mathrm{IL})-6$ levels $(n=6 ;(\mathbf{D}$ and $\mathbf{E}))$. ND represents normal diet mice; HFD represents HFD-induced DM mice. $* * P<0.01$. 


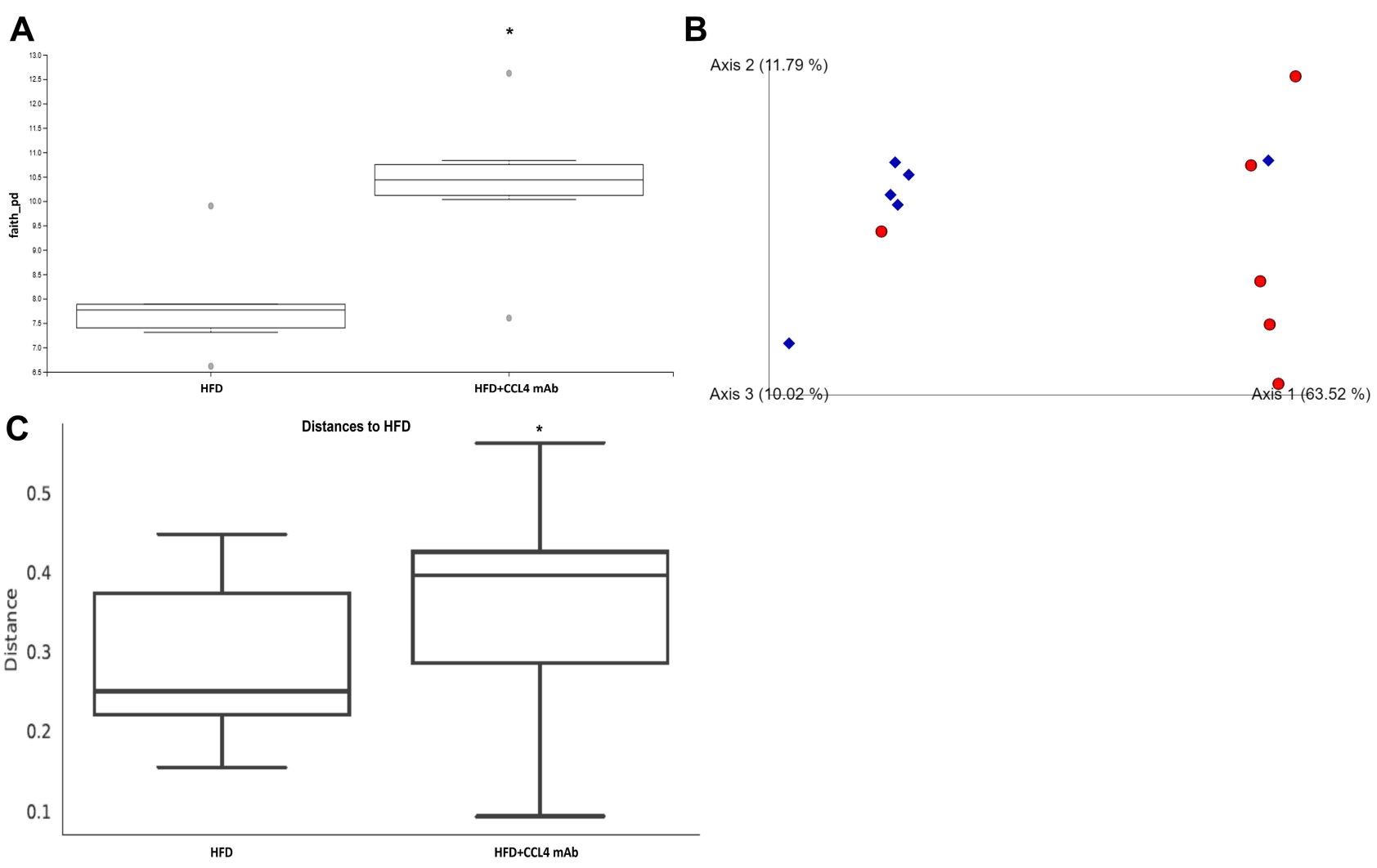

Figure 3 The effect of CCL4 inhibition on the structure of gut microbiota in HFD-induced DM mice. The $\alpha$-diversity of gut microbiota $(P=0.025 ; n=6 ;(A))$. Principal coordinate analysis (PCOA) of $\beta$-diversity based on unweighted UniFrac analysis. Samples collected from different groups are labeled with different shapes and colors. HFDinduced DM mice are shown as red circles; HFD-induced DM mice with CCL4 antibody injection for 4 weeks group are shown as blue diamonds $(n=6 ;(B))$. Composition difference analysis based on unweighted UniFrac analysis $(P=0.022 ; n=6 ;(C))$. HFD represents HFD-induced DM mice. *P $<0.05$.

has beneficial effects on glucose homeostasis, the lipid profile, and inflammation. We therefore investigated whether CCL4 inhibition led to specific alterations in the gut microbiota structure. The stool samples were analyzed for the microbial structure. The CCL4 inhibition group had a significant influence on the $\alpha$-diversity of the intestinal microbiota (Figure 3A). Mice that received CCL4 antibody for 4 weeks showed a distinct clustering of microbiota composition using principal coordinate analysis ( $\mathrm{PCoA})$ of $\beta$-diversity, which indicated that the composition of gut microbiota changed in response to CCL4 inhibition in HFD-induced DM mice (Figure 3B). In addition, the unweighted UniFrac distance analysis revealed that the differences in microbiota between groups were significant (Figure 3C).

The relative abundance of the predominant taxa examined by sequencing was analyzed to profile the specific changes in the gut microbiota (Figure 4). Taxon-based analysis revealed significant differences in gut microbial composition after CCL4 inhibition. The overall community patterns in the stool samples were altered after CCL4 inhibition at the phylum and family levels (Figure 4A and B). A significant increase of phylum Actinobacteria was observed in the CCL4 inhibition group (Figure 4A). At the family level, the relative abundance of Muribaculaceae was lower in the CCL4 inhibition group, while the relative abundance of Atopobiaceae was apparently higher in the CCL4 inhibition group than in untreated HFD-induced DM mice (Figure 4B). The ANCOM results indicated that the CCL4 inhibition group had a remarkable reduction of Muribaculaceae compared to the untreated HFD-induced DM mice (Figure 4C). These results showed that CCL4 inhibition could modulate the gut microbiota of HFD-fed mice, which may have caused the beneficial effects against HFD-induced obesity and DM.

The family tree and heatmap illustrated the presence of a different bacterial environment in the gut between the untreated HFD-induced DM group and CCL4 inhibition group (Figure 5A and B). Most importantly, CCL4 


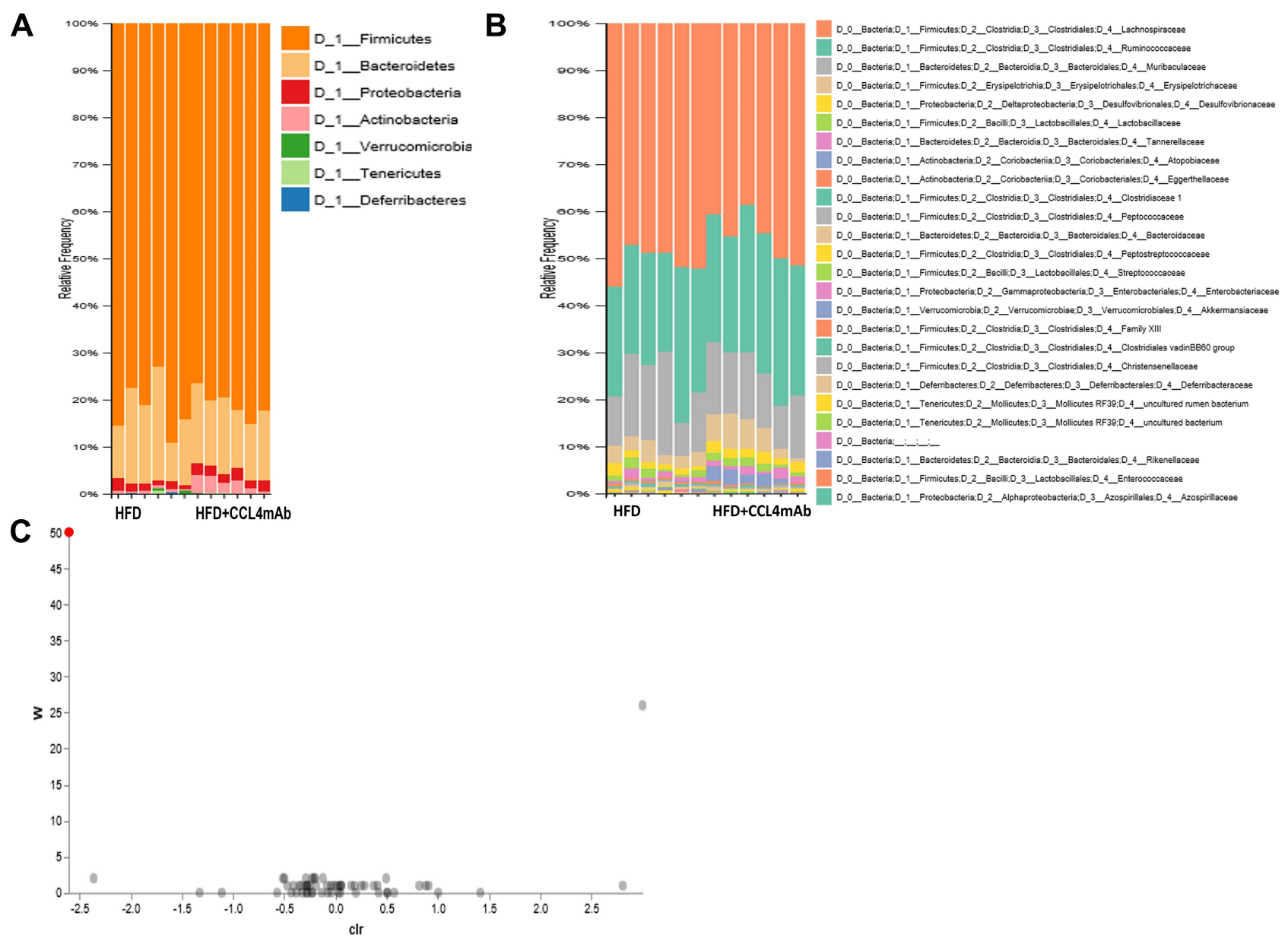

Figure 4 The effect of CCL4 inhibition on the composition of gut microbiota in HFD-induced DM mice. The relative abundances of dominant bacterial phyla $(n=6 ;(A))$ and families $(n=6$; (B)) in the HFD-induced DM group and HFD-induced DM mice with CCL4 antibody injection for 4 weeks group. ANCOM Volcano Plot of differential abundance. The red spot indicates family Muribaculaceae $(n=6 ;(\mathbf{C}))$. HFD represents HFD-induced DM mice.

inhibition could decrease the TMAO levels in HFDinduced DM mice (Figure 5C). In summary, CCL4 inhibition led to lower abundance of Muribaculaceae and higher abundance of Atopobiaceae at the family level, as well as a decreased concentration of TMAO.

\section{HFD-Induced DM Mice Received FMT from CCL4 Knockout Mice Had Improved Glucose Homeostasis}

CCL4 knockout mice were used to further confirm the pathological role of CCL4 in diet-induced DM. In the OGTT test, blood sugar levels were lower in the CCL4 knockout mice fed an HFD at 15 and 30 minutes than in the wild-type mice fed an HFD (Figure 6A). The AUCs of the OGTT were significantly lower in the CCL4 knockout mice fed an HFD than those in the wild-type mice fed an HFD (Figure 6B). The knockout mice fed an HFD also had lower levels of HOMA-IR than the wild-type mice fed an HFD (Figure 6C). These results indicated that CCL4 deficiency could truly improve glucose homeostasis in HFD-induced DM mice.

In another part of the study, we used FMT to verify the effect of CCL4 inhibition on gut microbiota and the linkage between CCL4-modulated gut microbiota and HFDinduced DM. In the OGTT test, blood sugar levels were lower in the wild-type mice fed an HFD with FMT from CCL4 knockout mice at 15 and 150 minutes than in those without FMT (Figure 6D). The AUCs of the OGTT were markedly lower in the wild-type mice fed an HFD with FMT from CCL4 knockout mice (Figure 6E). Moreover, the wild-type mice fed an HFD with FMT from CCL4 knockout mice had lower levels of HOMA-IR than those without FMT (Figure 6F). The above results definitely enhanced the causal relationship between CCL4 and the microbiota in the treatment of HFD-induced DM. 
A

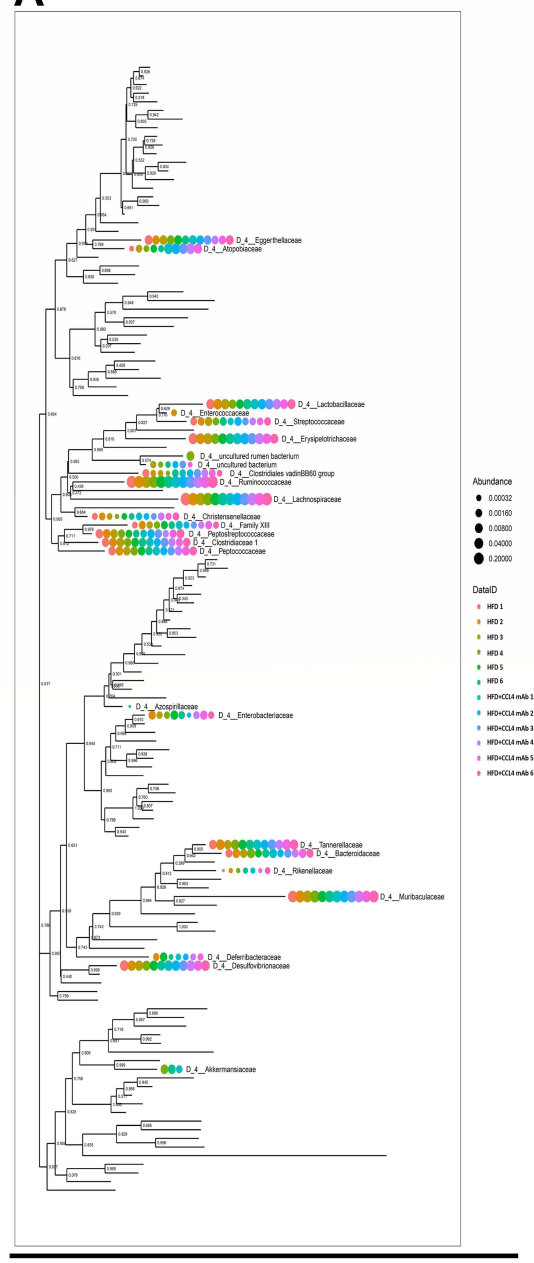

B
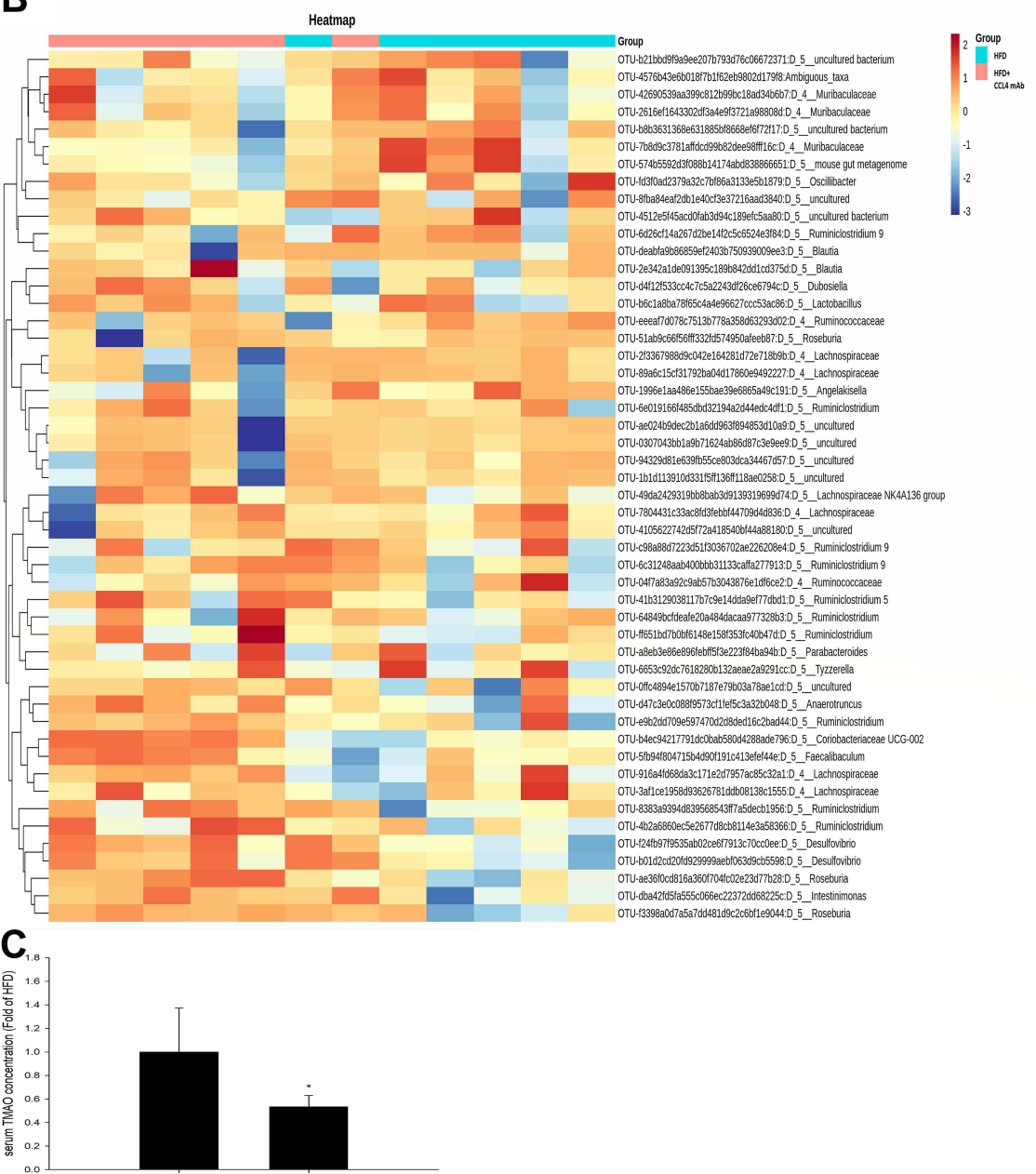

Figure 5 The effect of CCL4 inhibition on the gut microbiota and TMAO levels in HFD-induced DM mice. Family tree and heatmap (the relative abundances of 50 key taxa) analysis between gut microbiota and CCL4 inhibition in HFD-induced DM mice $(n=6 ;(\mathbf{A}$ and $\mathbf{B}))$. Serum levels of TMAO ( $n=6$; $(\mathbf{C})$. *P $<0.05)$.

\section{Discussion}

In this study, it was shown that CCL4 inhibition may alter the gut microbiota, and improve glucose metabolism, the lipid profile, and systemic inflammation in HFD-induced DM mice. Potential factors that may have contributed to the beneficial effects caused by CCL4 inhibition that we identified include a decreased relative abundance of family Muribaculaceae and an increased relative abundance of family Atopobiaceae in the gut microbiota, as well as a reduced circulating TMAO concentration. Given the suggested role of TMAO in systemic inflammation, these results indicated the potential association between the compositional changes in the gut microbiota and CCL4 inhibition-related indices in HFD-induced DM mice. More interestingly, FMT from CCL4-deficient donor could lead to protective efficacy on glucose homeostasis in HFDinduced DM, which support the potential effects of
CCL4 on gut microbiota for in vivo metabolic control. Given the limited number of experiment animals, the data may be preliminary. Future studies are indicated to investigate the complex role of gut microbiota, such as family Muribaculaceae and family Atopobiaceae in systemic inflammation as well as metabolic disorder.

There have been few previous studies related to the family Atopobiaceae, but there was a recently sequencing and cultivation study of the family Muribaculaceae. ${ }^{18}$ While the systemic role of the family Atopobiaceae is rarely known, the diverse impacts of the family Muribaculaceae was shown in different animal experiments.

Several types of animal models have been used in the experiments of gut microbiota for systemic metabolism. First, dietary supplements are usually used in this type of study, which may theoretically generate local intestinal 
A

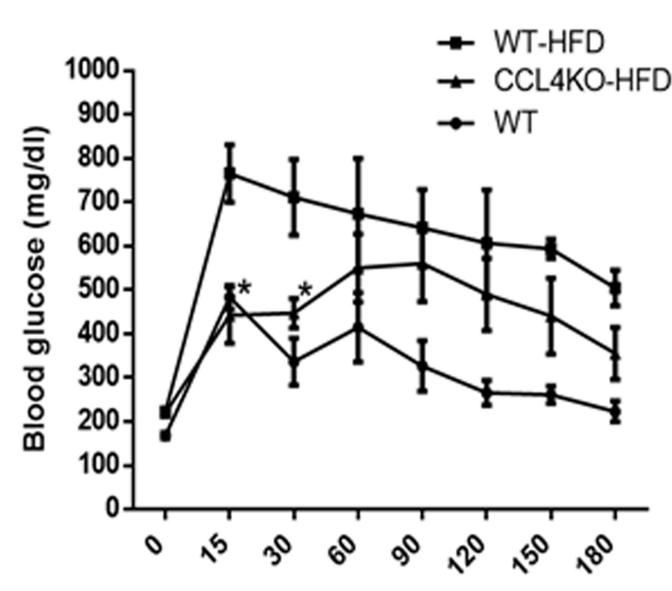

Time (minutes)

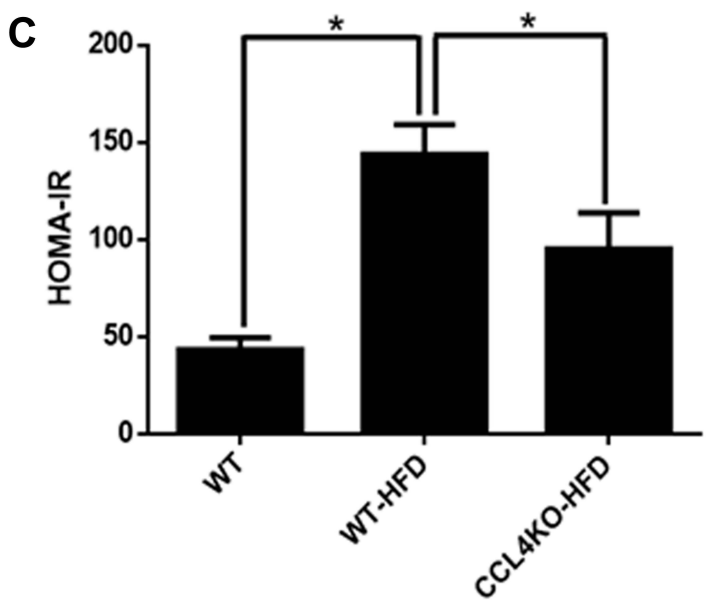

E

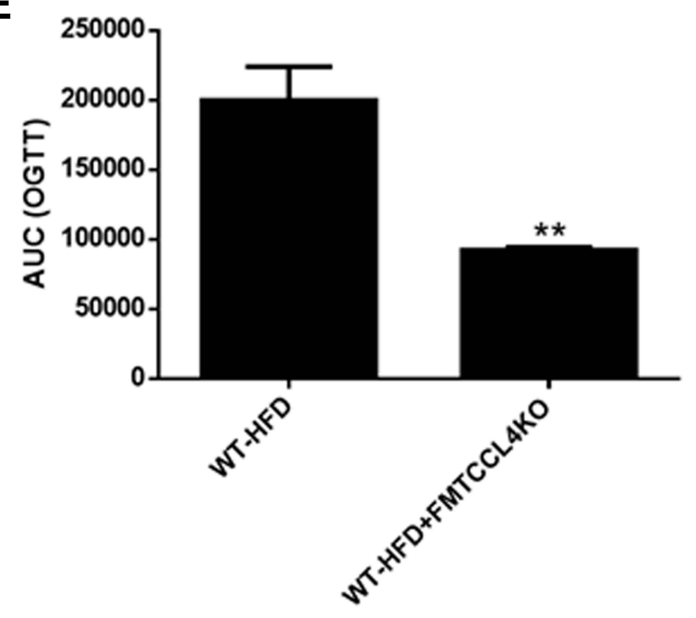

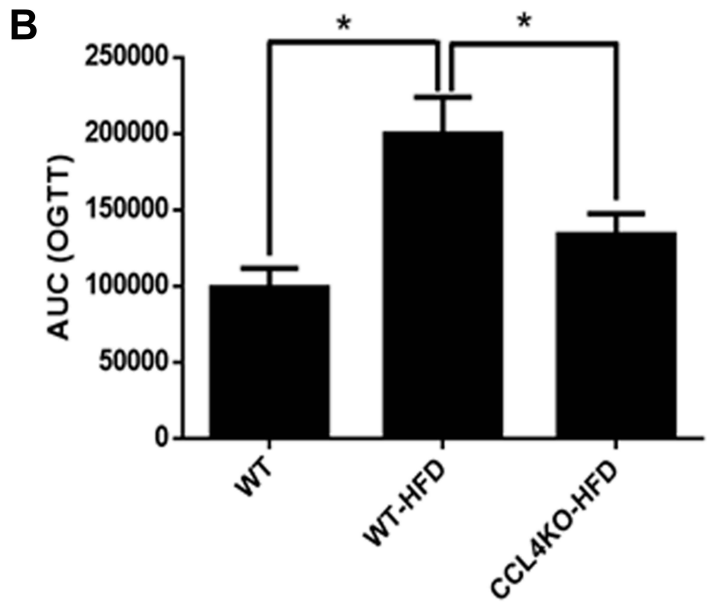

D

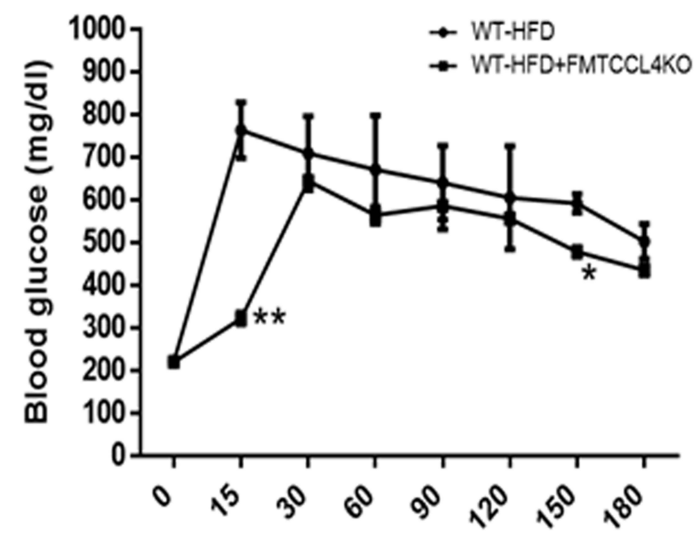

Time (minutes)

F

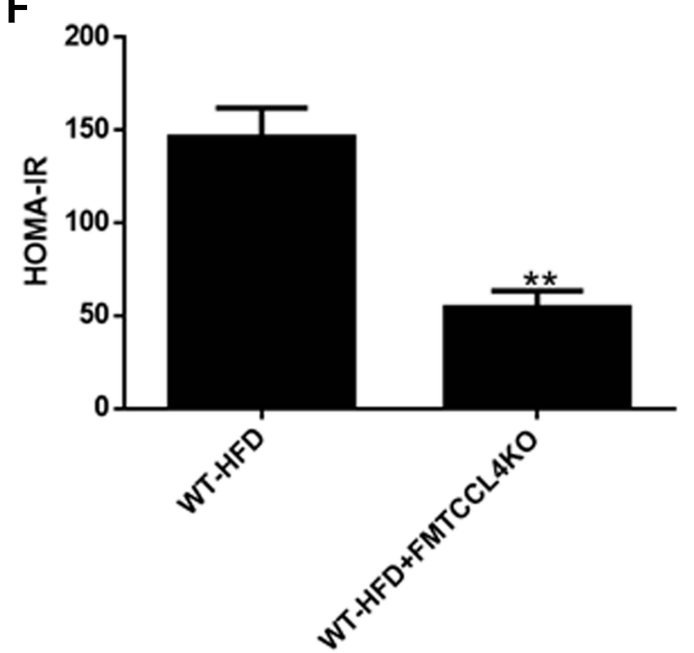

Figure 6 The effect of FMT from CCL4-deficient mice on glucose homeostasis in HFD-induced DM mice. The OGTTs, AUCs, and HOMA-IR were evaluated in both wildtype mice and CCL4 knockout mice fed an HFD $(n=6 ;(\mathbf{A}-\mathbf{C}))$. The OGTTs, AUCs, and HOMA-IR were confirmed in HFD-induced DM mice with or without the FMT from CCL4 knockout mice $(n=3$; $(\mathbf{D}-\mathbf{F}))$. WT represents wild-type mice; HFD represents HFD-induced DM mice; CCL4KO represents CCL4 knockout mice; FMT represents fecal microbiota transplant. $* \mathrm{P}<0.05, * * \mathrm{P}<0.01$. 
effects followed by systemic metabolic impacts. The oral administration of capsaicin was shown to reduce metabolic endotoxemia and chronic inflammation, which were associated with the anti-obesity effect and decreased abundance of the gut Muribaculaceae/S24-7 family. ${ }^{19}$ It was also shown that a polyphenol-rich extract of Dendrobium loddigesii could decrease the blood glucose, body weight, and low-density lipoprotein cholesterol levels, and increase the insulin levels in type 2 obese diabetic mice with decreased relative abundance of the family Muribaculaceae/S24-7. ${ }^{20}$ Another study showed that a raspberry extract, which may improve the severity of obesity-related symptoms, led to a decreased abundance of family Muribaculaceae/S24-7 family in the fecal microbiota of obese diabetic $\mathrm{db} / \mathrm{db}$ mice. ${ }^{21}$ On the other hands, the flower of Edgeworthia gardneri, a Chinese traditional medicine, could decrease the blood glucose level and ameliorate insulin resistance and lipid metabolism impairment in diabetic mice, as well as increase the family Muribaculaceae/S24-7 in the gut microbiota. ${ }^{22}$ Casein glycomacropeptide hydrolysate treatments reduced the concentration of LPS and pro-inflammatory cytokines in serum with enhanced abundance of gut Muribaculaceae/ S24-7 in streptozotocin-induced DM mice. ${ }^{23}$ Given the controversial findings in the dietary supplement animal models, other types of approach are required to clarify the specific role of Muribaculaceae/S24-7 in systemic metabolism.

Genetic modified mice may be another type of animal model that could be used for gut microbiota study. Recently, glutaredoxin 1-deficient mice was shown to exert upregulated lipid homeostasis biomarkers and Nrf2/ Keap1 expression, as well as antioxidant defense genes with a lower abundance of Muribaculaceae/S24-7, which may suggest the association of suppressed family Muribaculaceae/S24-7 with favorable metabolic status. ${ }^{24}$ In such case, both systemic inflammation and gut microbiota might be modified individually and simultaneously due to the general genetic background.

In the present study, systemic CCL4 inhibition, another novel experiment approach, improves glucose metabolism and lipid profiles with the downregulation of family Muribaculaceae and upregulation of family Atopobiaceae in the gut microbiota of HFD-induced DM mice. It has been indicated that inflammatory mediators including TNF$\alpha$ and IL- 6 are increased with insulin resistance and play an important role in deregulating glucose homeostasis in metabolic disorders. ${ }^{25}$ While TNF- $\alpha$ and IL- 6 were used as the major markers in the current study, other inflammatory biomarkers such as IL-1 $\beta, \mathrm{MCP} 1$, and so on may be also examined for a more comprehensive inflammatory profile of CCL4 inhibition in the future study. In the present study, both inflammatory proteins such as TNF- $\alpha$ and IL- 6 and circulating TAMO level as a pro-inflammatory metabolite from gut microbiota were reduced by direct CCL4 inhibition. It seems that the systemic effects of CCL4 inhibition might alter the gut microbiota via a unique pathway, which may then improve the microbiota profiles for a less inflammatory status evident by the reduced circulating TAMO level. Furthermore, the lipid profiles were also improved by CCL4 antibodies in HFD-induced DM mice in the current study. Accordingly, the lipid profiles of CCL4 knockout mice and HFD-induced DM mice receiving FMT from CCL4 knockout mice should be interesting. While the lipid profiles were not examined in DM mice receiving FMT from CCL4 knockout mice in the current study, future studies may be required to further elucidate the detailed mechanisms of CCL4-related inflammation in systemic metabolism of both glucose and lipids.

TMAO is a metabolite that has attracted attention because of its close association with several chronic inflammatory diseases such as type $2 \mathrm{DM}$, atherosclerosis, and chronic kidney disease. ${ }^{26}$ The production of TMAO results from the conversion by the gut microbiota of dietary nutrients. Specifically, choline and carnitine can be transformed to trimethylamine and converted into TMAO in the liver by flavin-containing monooxygenase 1 and 3. Studies have shown that nutrients and bioactive compounds from food can modulate the gut microbiota to reduce TMAO production. ${ }^{27}$ HFD-induced obese NLRP3-deficient mice had an altered composition of gut microbiota associated with reduced levels of TMAO and LPS, attenuated systemic inflammation, and improved lipid metabolism. ${ }^{28}$ Given that higher levels of circulating TMAO could be associated with a higher risk of $\mathrm{DM},{ }^{29}$ it is then important to develop the novel interventional strategy that manipulates the production of TMAO. Interestingly, in the present study, we did observe that CCL4 inhibition could reduce the levels of TMAO with a significant and probably favorable alteration of the gut microbiota.

Accordingly, our findings might support the potential pathogenic role of family Muribaculaceae suggested by previous studies. While the bio-effects of family Atopobiaceae are scanty known, family Muribaculaceae/ S24-7 is found as an LPS-producing bacterium related to LPS biosynthesis. It has been shown that in HFD-fed 
mice, the modulation of gut microbiota was linked to an increased intestinal permeability and LPS absorption that amplified the development of inflammation and metabolic disorders. ${ }^{30}$ A previous study found that the bacteriumassociated LPS could bind to toll-like receptor-4 and led to increased levels of proinflammatory cytokines. ${ }^{31}$ In mice fed a high-fat diet, an enhanced abundance of the Gramnegative S24-7 family was observed, and chronic lowgrade inflammation was prevented with lower levels of the family S24-7. ${ }^{19}$ Decreased levels of Muribaculaceae/ S24-7 in the gut microbiota were also associated with antiinflammatory effects from decreasing the expression of TNF- $\alpha$ and IL-6 in diabetic db/db mice. ${ }^{20}$ In this study, we further showed that in a high-fat diet-induced diabetic mouse model, direct systemic CCL4 inhibition could result in suppressed gut microbiota Muribaculaceae/S24-7, decreased circulating TMAO level, and reduced systemic inflammatory proteins, such as TNF- $\alpha$ and IL-6. Taken together, one may speculate the complex effects of direct CCL4 inhibition in vivo. In addition to modulating circulating inflammatory cells and pancreatic inflammation by direct inhibition on CCL4, ${ }^{14}$ there might be a novel local mechanism of CCL4 inhibition to modulate gut microbiota particularly family Muribaculaceae/S24-7 and family Atopobiaceae, which then reduce LPS related, toll-like receptor-4 and Nrf2/Keap1-mediated production of inflammatory proteins. While our findings support the hypothesis that direct CCL4 inhibition could modify gut microbiota and related systemic metabolism in diet-induced DM mice, further investigations are required to elucidate the CCL4related signaling pathways in gut.

On the other hand, though less likely, it is possible that direct inhibition on CCL4 may alter systemic inflammation that leads to changes in gut microbiota. In such case, gut microbiota profiles, similar to circulating C-reactive protein levels, may be just a mirror to the status of systemic inflammation. While the family Muribaculaceae has been suggested pathogenic and linked to systemic inflammation and metabolic disorder, our findings of the modulation on family Muribaculaceae by direct CCL4 inhibition seem meaningful. More interestingly, the specific pathology of HFD-induced DM could be potentially rescued by CCL4-mediated gut microbiota through the FMT experiments.

There are some concerns about the current study that should be further indicated. In our study, an FMT mouse model with CCL4 knockout mice was used to validate the beneficial effects of CCL4 inhibition in HFD-induced DM. While the fecal from CCL4 knockout mice would not be equal to the mouse fecal after CCL4 antibody treatment, a sequencing of stool from CCL4 knockout mice might be needed for further evaluation. On the other hand, a previous study compared the depletion of gut microbes by administering a single broad-spectrum antibiotic and indicated that each option individually elicited unique taxonomic changes. ${ }^{32}$ In the current study, antibiotic water containing ciprofloxacin was used to create a control environment in gut for FMT. While a combination of antibiotics was frequently used in previous FMT experiments, it is interesting to see if our current observations may be validated in another FMT model with the antibiotic cocktail. Finally, for advanced mechanistic insight, further studies are ongoing to elucidate the direct impact of gut microbiota including family Muribaculaceae and family Atopobiaceae on systemic inflammation that related to in vivo glucose metabolism.

\section{Conclusion}

Direct CCL4 inhibition altered gut microbiota profiles, with reduced abundance of family Muribaculaceae, enhanced abundance of family Atopobiaceae, and decreased TMAO production in vivo. The above changes were associated with the reduction of systemic inflammation and the improvements of glucose as well as lipid metabolism in HFD-induced DM mice. In line with the inflammatory theory of glucose metabolic disorders, our findings highlight the significant impacts of CCL4 on both systemic inflammation, metabolism, and gut microbiota. Furthermore, FMT from CCL4 knockout mice could potentially rescue the glucose homeostasis in HFDinduced DM mice. Given the established role of gut microbiota in systemic inflammation and metabolism, our findings could provide a novel and important rationale to CCL4-based approach in diet-induced DM. Theoretically, they may have potential clinical impacts, particularly in chronic systemic inflammation with elevated CCL4 levels such as type 2 DM and atherosclerosis disease. While the current findings might be observational, additional studies are ongoing to investigate the direct role of family Muribaculaceae and family Atopobiaceae as the potential modifiable targets for metabolic control in either experimental or clinical DM.

\section{Funding}

This work was supported by the Taipei Veterans General Hospital, Taipei, Taiwan [V110C-065, V109C-052, and V108D41-004-MY3] and Ministry of Science and Technology, Taipei, Taiwan [MOST 110-2314-B-075-058MY3]. 


\section{Disclosure}

The authors report no conflicts of interest in this work.

\section{References}

1. Tilg H, Moschen AR. Microbiota and diabetes: an evolving relationship. Gut. 2014;63:1513-1521. doi:10.1136/gutjnl-2014306928

2. Jie Z, Xia H, Zhong SL, et al. The gut microbiome in atherosclerotic cardiovascular disease. Nat Commun. 2017;8:845. doi:10.1038/ s41467-017-00900-1

3. Croci S, D'Apolito LI, Gasperi V, et al. Dietary strategies for management of metabolic syndrome: role of gut microbiota metabolites. Nutrients. 2021;13:1389. doi:10.3390/nu13051389

4. Akash MS, Rehman K, Chen S. Role of inflammatory mechanisms in pathogenesis of type 2 diabetes mellitus. $J$ Cell Biochem. 2013;114:525-531. doi:10.1002/jcb.24402

5. Abdel-Moneim A, Bakery HH, Allam G. The potential pathogenic role of IL-17/Th17 cells in both type 1 and type 2 diabetes mellitus. Biomed Pharmacother. 2018;101:287-292. doi:10.1016/j.biopha.20 18.02.103

6. Kau AL, Ahern PP, Griffin NW, et al. Human nutrition, the gut microbiome and the immune system. Nature. 2011;474:327-336. doi:10.1038/nature 10213

7. Moschen AR, Wieser V, Tilg H. Dietary factors: major regulators of the gut's microbiota. Gut Liver. 2012;6:411-416. doi:10.5009/ gn1.2012.6.4.411

8. Shin NR, Lee JC, Lee HY, et al. An increase in the Akkermansia spp. population induced by metformin treatment improves glucose homeostasis in diet-induced obese mice. Gut. 2014;63:727-735. doi:10. 1136/gutjnl-2012-303839

9. Li Y, Cui Y, Hu X, et al. Chlorophyll supplementation in early life prevents diet-induced obesity and modulates gut microbiota in mice. Mol Nutr Food Res. 2019;63:e1801219. doi:10.1002/ mnfr.201801219

10. Pham MN, Hawa MI, Roden M, et al. Increased serum concentrations of adhesion molecules but not of chemokines in patients with Type 2 diabetes compared with patients with Type 1 diabetes and latent autoimmune diabetes in adult age: action LADA 5. Diabet Med. 2012;29:470-478. doi:10.1111/j.1464-5491.2011.03546.x

11. Chang TT, Chen JW. Emerging role of chemokine CC motif ligand 4 related mechanisms in diabetes mellitus and cardiovascular disease: friends or foes? Cardiovasc Diabetol. 2016;15:117. doi:10.1186/ s12933-016-0439-9

12. Lee Y, Kwon EY, Choi MS. Dietary isoliquiritigenin at a low dose ameliorates insulin resistance and NAFLD in diet-induced obesity in C57BL/6J mice. Int J Mol Sci. 2018;19:3281. doi:10.3390/ijms 19103281

13. Jahns L, Conrad Z, Johnson LK, et al. A diet high in carotenoid-rich vegetables and fruits favorably impacts inflammation status by increasing plasma concentrations of IFN-alpha2 and decreasing MIP-1beta and TNF-alpha in healthy individuals during a controlled feeding trial. Nutr Res (New York, NY). 2018;52:98-104. doi:10.1016/j.nutres.2018.02.005

14. Chang TT, Lin LY, Chen JW. A novel resolution of diabetes: C-C chemokine motif ligand 4 is a common target in different types of diabetes by protecting pancreatic islet cell and modulating inflammation. Front Immunol. 2021;12:650626. doi:10.3389/fimmu. 2021.650626

15. Maugham ML, Thomas PB, Crisp GJ, et al. Insights from engraftable immunodeficient mouse models of hyperinsulinaemia. Sci Rep. 2017;7:491. doi:10.1038/s41598-017-00443-x
16. Friedewald WT, Levy RI, Fredrickson DS. Estimation of the concentration of low-density lipoprotein cholesterol in plasma, without use of the preparative ultracentrifuge. Clin Chem. 1972;18:499-502. doi:10.1093/clinchem/18.6.499

17. Cani PD, Amar J, Iglesias MA, et al. Metabolic endotoxemia initiates obesity and insulin resistance. Diabetes. 2007;56:1761-1772. doi:10.2337/db06-1491

18. Lagkouvardos I, Lesker TR, Hitch TCA, et al. Sequence and cultivation study of Muribaculaceae reveals novel species, host preference, and functional potential of this yet undescribed family. Microbiome. 2019;7:28. doi:10.1186/s40168-019-0637-2

19. Kang C, Wang B, Kaliannan K, et al. Gut microbiota mediates the protective effects of dietary capsaicin against chronic low-grade inflammation and associated obesity induced by high-fat diet. mBio. 2017;8:e00470-17.

20. Li XW, Chen HP, He YY, et al. Effects of rich-polyphenols extract of dendrobium loddigesii on anti-diabetic, anti-inflammatory, anti-oxidant, and gut microbiota modulation in $\mathrm{db} / \mathrm{db}$ mice. Molecules (Basel, Switzerland). 2018;23:3245. doi:10.3390/ molecules 23123245

21. Garcia-Mazcorro JF, Pedreschi R, Chew B, et al. Dietary supplementation with raspberry extracts modifies the fecal microbiota in obese diabetic db/db mice. J Microbiol Biotechnol. 2018;28:1247-1259. doi:10.4014/jmb.1803.03020

22. Zhang Z, Xu H, Zhao H, et al. Edgeworthia gardneri (Wall.) Meisn. water extract improves diabetes and modulates gut microbiota. J Ethnopharmacol. 2019;239:111854. doi:10.1016/j.jep.2019.111854

23. Yuan Q, Zhan B, Chang R, et al. Antidiabetic effect of casein glycomacropeptide hydrolysates on high-fat diet and STZ-induced diabetic mice via regulating insulin signaling in skeletal muscle and modulating gut microbiota. Nutrients. 2020;12:220. doi:10.3390/nu12010220

24. Ahmad MI, Zou X, Ijaz MU, et al. Processed meat protein promoted inflammation and hepatic lipogenesis by upregulating Nrf2/Keap1 signaling pathway in Glrx-deficient mice. J Agric Food Chem. 2019;67:8794-8809. doi:10.1021/acs.jafc.9b03136

25. De Felice FG, Ferreira ST. Inflammation, defective insulin signaling, and mitochondrial dysfunction as common molecular denominators connecting type 2 diabetes to Alzheimer disease. Diabetes. 2014;63:2262-2272. doi:10.2337/db13-1954

26. Wang Z, Klipfell E, Bennett BJ, et al. Gut flora metabolism of phosphatidylcholine promotes cardiovascular disease. Nature. 2011;472:57-63. doi:10.1038/nature09922

27. Coutinho-Wolino KS, de FC, de Oliveira Leal V, et al. Can diet modulate trimethylamine N-oxide (TMAO) production? What do we know so far? Eur J Nutr. 2021;60:3567-3584. doi:10.1007/ s00394-021-02491-6

28. Sokolova M, Yang K, Hansen SH, et al. NLRP3 inflammasome deficiency attenuates metabolic disturbances involving alterations in the gut microbial profile in mice exposed to high fat diet. Sci Rep. 2020;10:21006. doi:10.1038/s41598-020-76497-1

29. Zhuang R, Ge X, Han L, et al. Gut microbe-generated metabolite trimethylamine $\mathrm{N}$-oxide and the risk of diabetes: a systematic review and dose-response meta-analysis. Obes Rev. 2019;20:883-894. doi:10.1111/obr.12843

30. Cani PD, Bibiloni R, Knauf C, et al. Changes in gut microbiota control metabolic endotoxemia-induced inflammation in high-fat diet-induced obesity and diabetes in mice. Diabetes. 2008;57: 1470-1481. doi:10.2337/db07-1403

31. Caesar R, Tremaroli V, Kovatcheva-Datchary P, et al. Crosstalk between gut microbiota and dietary lipids aggravates WAT inflammation through TLR signaling. Cell Metab. 2015;22:658-668. doi:10. 1016/j.cmet.2015.07.026

32. Cabral DJ, Penumutchu S, Reinhart EM, et al. Microbial metabolism modulates antibiotic susceptibility within the murine gut microbiome. Cell Metab. 2019;30:800-823.e807. doi:10.1016/j.cmet.2019.08.020 


\section{Publish your work in this journal}

The Journal of Inflammation Research is an international, peerreviewed open-access journal that welcomes laboratory and clinical findings on the molecular basis, cell biology and pharmacology of inflammation including original research, reviews, symposium reports, hypothesis formation and commentaries on: acute/chronic inflammation; mediators of inflammation; cellular processes; molecular mechanisms; pharmacology and novel anti-inflammatory drugs; clinical conditions involving inflammation. The manuscript management system is completely online and includes a very quick and fair peerreview system. Visit http://www.dovepress.com/testimonials.php to read real quotes from published authors. 